\title{
Early Recurrence in Completely Resected IIIB and IIIC Melanoma Warrants Restaging Prior to Adjuvant Therapy
}

\author{
Martine Bloemendal, $\mathrm{MD}^{1,2}$, Wouter W. van Willigen, $\mathrm{MD}^{1,2}$, Kalijn F. Bol, MD, $\mathrm{PhD}^{1,2}$, \\ Marye J. Boers-Sonderen, MD, $\mathrm{PhD}^{1}$, Johannes J. Bonenkamp, MD, $\mathrm{PhD}^{3}$, J. E. M. Werner, $\mathrm{MD}^{3}$, \\ Erik H. J. G. Aarntzen, MD, $\mathrm{PhD}^{4}$, Rutger H. T. Koornstra, MD, $\mathrm{PhD}^{1}$, Jan Willem B. de Groot, $\mathrm{MD}$, $\mathbf{P h D}^{5}$, \\ I. Jolanda M. de Vries, PhD $^{2}$, Jacobus J. M. van der Hoeven, MD, PhD $^{1}$, Winald R. Gerritsen, MD, PhD ${ }^{1}$, and \\ Johannes H. W. de Wilt, $\mathrm{MD}, \mathrm{PhD}^{3}$ \\ ${ }^{1}$ Department of Medical Oncology, Radboud University Medical Center, Nijmegen, The Netherlands; ${ }^{2}$ Department of \\ Tumor Immunology, Radboud Institute for Molecular Life Sciences, Nijmegen, The Netherlands; ${ }^{3}$ Department of Surgery, \\ Radboud University Medical Center, Nijmegen, The Netherlands; ${ }^{4}$ Department of Radiology and Nuclear Medicine, \\ Radboud University Medical Center, Nijmegen, The Netherlands; ${ }^{5}$ Oncological Center Isala, Zwolle, The Netherlands
}

\begin{abstract}
Purpose. To evaluate the results of restaging completely resected stage IIIB/C melanoma prior to start of adjuvant therapy.

Patients and Methods. One hundred twenty patients with stage IIIB or IIIC (AJCC 2009) melanoma who underwent complete surgical resection were screened for inclusion in our trial investigating adjuvant dendritic cell therapy (NCT02993315). All patients underwent imaging to exclude local relapse or metastasis before entering the trial. The frequency of recurrent disease within 12 weeks after resection and the method of detection were investigated.

Results. Sixty-nine (58\%) stage IIIB and $51(43 \%)$ stage IIIC melanoma patients were screened. Median age was 54 (range 27-79) years. Twenty-two (18\%) of 120 patients with completely resected stage IIIB/C melanoma had evidence of early recurrent disease, despite exclusion thereof by prior imaging. Median interval between resection and detection of relapse was 7.4 (range 4.3-10.7) weeks. Recurrence was asymptomatic in 17 (77\%) patients, but metastasis was noticed by the patient or physician in 5
\end{abstract}

Wouter W. van Willigen and Kalijn F. Bol have contributed equally to this work.

(C) The Author(s) 2019

First Received: 19 December 2018;

Published Online: 4 March 2019

J. H. W. de Wilt, MD, PhD

e-mail: hans.dewilt@radboudumc.nl
(23\%). Eight patients with local relapse received local treatment with curative intent, and one was treated with systemic therapy. The remaining patients had distant metastasis, 1 of whom underwent resection of a solitary liver metastasis while 12 patients received systemic treatment.

Conclusions. Patients with completely resected stage IIIB/ $\mathrm{C}$ melanoma have high risk of early recurrence before start of adjuvant therapy. Restaging should be considered for high-risk melanoma patients before start of adjuvant therapy.

Treatment of stage III melanoma consists of complete resection with curative intent. However, the risk of recurrence afterwards is high, resulting in 5-year overall survival (OS) rates between 40 and $78 \% .^{1-3}$ Therapeutic options and prospects for patients with metastatic melanoma have changed considerably in recent years, especially with the introduction of immune checkpoint inhibitors and BRAF and MEK inhibitors. ${ }^{4-10}$ These drugs have been proven to significantly improve OS in metastatic melanoma and have also shown promising results in the adjuvant setting. Phase III trials investigating adjuvant systemic therapy with ipilimumab (anti-CTLA-4 antibody) and combined dabrafenib/trametinib (BRAF/MEK-inhibitor) showed improved OS compared with placebo. ${ }^{11,12}$ Adjuvant nivolumab and pembrolizumab (both anti-PD-1 antibodies) led to improved 12-month recurrence-free survival (RFS) rates when compared with ipilimumab and placebo, respectively. ${ }^{13,14}$ Data on OS are still awaited. These results led 
to approval of ipilimumab, pembrolizumab, nivolumab, and combined dabrafenib/trametinib as adjuvant therapy by the Food and Drug Administration (FDA). The European Medicines Agency (EMA) approved use of nivolumab and combined dabrafenib/trametinib in the adjuvant setting and received a positive advice from the Committee for Medicinal Products for Human Use (CMHP) for adjuvant use of pembrolizumab. ${ }^{15-23}$

After diagnosis of nodal metastasis in high-risk stage III melanoma, imaging techniques [e.g. computed tomography (CT) or ${ }^{18} \mathrm{~F}$-fluorodeoxyglucose $\left({ }^{18} \mathrm{~F}\right.$-FDG) positron emission tomography (PET)] are used to exclude distant metastasis. In stage IIIB/C melanoma, most recurrences appear within the first 2 years after surgical resection. ${ }^{1}$ Despite this high risk, incorporation of imaging techniques in follow-up after resection differs widely between centers. No survival benefit of imaging during follow-up was demonstrated in a randomized trial, but this was carried out prior to the introduction of effective therapies for metastatic melanoma. ${ }^{24,25}$ In a clinical trial investigating adjuvant therapy, it is mandatory to exclude recurrent disease prior to inclusion, preventing metastatic melanoma patients from entering the adjuvant study.

We report herein imaging results for 120 stage IIIB and IIIC melanoma patients who underwent complete surgical resection within 12 weeks prior to inclusion in a placebocontrolled, randomized trial investigating adjuvant dendritic cell therapy (NCT02993315). Imaging with contrastenhanced venous-phase CT (ceCT) or ${ }^{18}$ F-FDG PET/CT was performed to exclude recurrent disease within 6 weeks prior to inclusion.

\section{PATIENTS AND METHODS}

\section{Patients}

After signing informed consent, patients were screened for eligibility in a placebo-controlled randomized trial (NCT02993315) investigating adjuvant dendritic cell vaccination. The protocol has been approved by the national review committee (Central Committee on Research Involving Human Subjects) and is in concordance with the Declaration of Helsinki and Good Clinical Practice. Eligible patients were adults with stage IIIB or IIIC [American Joint Committee on Cancer (AJCC) 7th edition $]^{2}$ cutaneous melanoma within 12 weeks after complete radical lymph node dissection (RLND) and after recovery from the surgery. The protocol was amended after publication of the MSLT-II trial results, which showed no survival benefit of completion lymph node dissection after removal of microscopic metastasis with sentinel node biopsy (SNB) when compared with nodal surveillance. ${ }^{26}$
After amendment, patients with microscopic disease could be included after SNB and additional completion lymph node dissection was no longer required. Macrometastasis was defined as a palpable node or as a nonpalpable node of at least $15 \mathrm{~mm}$ in short axis on CT, a PET-positive node, or one or more foci of melanoma of at least $1 \mathrm{~cm}$ in diameter in the pathology report. Patients with completely resected in-transit and/or satellite metastasis, an unknown primary tumor, and (planned) adjuvant radiotherapy could be included. In addition, absence of distant metastasis had to be documented by ceCT of the chest, abdomen, and pelvis or whole-body ${ }^{18} \mathrm{~F}$-FDG PET scan combined with CT $\left({ }^{18} \mathrm{~F}\right.$ FDG PET/CT) within 6 weeks before inclusion in our trial. In patients with head or neck melanoma, additional ceCT of the neck was obligatory. Imaging of the brain was performed in case of clinical suspicion of brain metastasis. Exclusion criteria included autoimmune disease (except for skin disease, hypothyroidism after autoimmune thyroiditis, and type 1 diabetes mellitus), a second malignancy in the last 5 years (except for adequately treated carcinoma in situ and basal or squamous cell carcinoma of the skin), concomitant use of oral or intravenous immunosuppressive drugs, and uncontrolled infectious disease.

\section{METHODS}

Within 6 weeks prior to the start of the study, imaging to exclude relapse was performed. Recurrence was considered symptomatic if suspected by symptoms and/or abnormalities during physical examination. Otherwise, recurrence was considered asymptomatic. Blood tests, including lactate dehydrogenase (LDH), were carried out within 4 weeks before inclusion. For baseline characteristics, a conglomerate of lymph nodes with at least four metastatic lymph nodes and presence of extracapsular extension was regarded as N3 disease. In case of a conglomerate, the diameter of lymph node involvement was counted as the diameter of the conglomerate.

\section{RESULTS}

\section{Patient Characteristics}

Between November 2016 and July 2018, 120 patients were screened for eligibility. Baseline characteristics are presented in Table 1. Median age was 54 (range 27-79) years, and $76(63 \%)$ of patients were male. Sixty-nine $(58 \%)$ and $51(43 \%)$ patients were diagnosed with stage IIIB and IIIC melanoma, respectively. Twenty-one (18\%) patients had completely resected in-transit metastasis, and nine $(8 \%)$ patients presented with nodal metastasis from an unknown primary tumor. Baseline characteristics of 
TABLE 1 Baseline characteristics

\begin{tabular}{|c|c|c|c|}
\hline Characteristic & $\begin{array}{l}\text { Total } \\
(n=120)\end{array}$ & $\begin{array}{l}\text { No recurrent disease } \\
\text { during screening }(n=98)\end{array}$ & $\begin{array}{l}\text { Recurrent disease } \\
\text { during screening }(n=22)\end{array}$ \\
\hline Median (range) age (years) & $54(27-79)$ & $55(27-79)$ & $51(27-73)$ \\
\hline \multicolumn{4}{|l|}{ Sex, $n(\%)$} \\
\hline Male & $76(63)$ & $59(60)$ & $17(77)$ \\
\hline Female & $44(37)$ & $39(40)$ & $5(23)$ \\
\hline \multicolumn{4}{|l|}{ Stage at screening (AJCC 7th edition), $n(\%)$} \\
\hline IIIB & $69(58)$ & $58(59)$ & $11(50)$ \\
\hline IIIC & $51(43)$ & $40(41)$ & $11(50)$ \\
\hline \multicolumn{4}{|l|}{ Breslow, $n(\%)^{\mathrm{a}}$} \\
\hline$<2 \mathrm{~mm}$ & $49(44)$ & $42(47)$ & $7(32)$ \\
\hline $2-4 \mathrm{~mm}$ & $24(22)$ & $19(21)$ & $5(23)$ \\
\hline$\geq 4 \mathrm{~mm}$ & $36(32)$ & $27(30)$ & $9(41)$ \\
\hline Other $^{\mathrm{b}}$ & $2(2)$ & $1(1)$ & $1(5)$ \\
\hline \multicolumn{4}{|l|}{ Ulceration, $n(\%)^{\mathrm{a}}$} \\
\hline Yes & $38(32)$ & $31(32)$ & $7(32)$ \\
\hline No & $73(61)$ & $58(59)$ & $15(68)$ \\
\hline \multicolumn{4}{|l|}{ Histological type, $n(\%)^{\mathrm{a}}$} \\
\hline Superficial spreading melanoma & $73(66)$ & $61(69)$ & $12(55)$ \\
\hline Nodular melanoma & $26(23)$ & $20(22)$ & $6(27)$ \\
\hline Other & $7(6)$ & $5(6)$ & $2(9)$ \\
\hline Missing & $5(5)$ & $3(3)$ & $2(9)$ \\
\hline \multicolumn{4}{|l|}{ Primary site, $n(\%)$} \\
\hline Head/neck & $17(14)$ & $13(13)$ & $4(18)$ \\
\hline Trunk & $46(38)$ & $37(38)$ & $9(41)$ \\
\hline Upper extremity & $13(11)$ & $12(12)$ & $1(5)$ \\
\hline Lower extremity & $34(28)$ & $26(27)$ & $8(36)$ \\
\hline Genital & $1(1)$ & $1(1)$ & $0(0)$ \\
\hline Unknown primary & $9(8)$ & $9(9)$ & $0(0)$ \\
\hline \multicolumn{4}{|l|}{ Type of lymph node involvement, $n(\%)$} \\
\hline Microscopic & $21(18)$ & $19(19)$ & $2(9)$ \\
\hline Macroscopic & $99(83)$ & $79(81)$ & $20(91)$ \\
\hline Median (range) maximum diameter of lymph node metastasis $(\mathrm{cm})$ & $2.0(0.01-7.5)$ & $1.9(0.01-7.5)$ & $3.0(0.25-7.0)$ \\
\hline \multicolumn{4}{|l|}{ Number of metastatic lymph nodes, $n(\%)$} \\
\hline 0 & $1(1)$ & $1(1)$ & $0(0)$ \\
\hline 1 & $46(38)$ & $40(41)$ & $6(27)$ \\
\hline $2-3$ & $37(31)$ & $29(30)$ & $8(36)$ \\
\hline$\geq 4$ & $36(30)$ & $28(29)$ & $8(36)$ \\
\hline \multicolumn{4}{|l|}{ Site of nodal metastasis } \\
\hline Neck & $26(22)$ & $21(21)$ & $5(23)$ \\
\hline Axilla & $51(43)$ & $46(47)$ & $5(23)$ \\
\hline Groin & $42(35)$ & $30(31)$ & $12(55)$ \\
\hline Popliteal & $1(1)$ & $1(1)$ & $0(0)$ \\
\hline \multicolumn{4}{|l|}{ Extracapsular extension, $n(\%)$} \\
\hline Yes & $30(25)$ & $23(23)$ & $7(32)$ \\
\hline No & $67(56)$ & $56(57)$ & $11(50)$ \\
\hline Missing & $23(19)$ & $19(19)$ & $4(18)$ \\
\hline
\end{tabular}


TABLE 1 continued

\begin{tabular}{|c|c|c|c|}
\hline Characteristic & $\begin{array}{l}\text { Total } \\
(n=120)\end{array}$ & $\begin{array}{l}\text { No recurrent disease } \\
\text { during screening }(n=98)\end{array}$ & $\begin{array}{l}\text { Recurrent disease } \\
\text { during screening }(n=22)\end{array}$ \\
\hline \multicolumn{4}{|c|}{ In-transit or (micro)satellite metastases, $n(\%)^{\mathrm{c}}$} \\
\hline Yes & $21(18)$ & $17(17)$ & $4(18)$ \\
\hline No & $99(83)$ & $81(83)$ & $18(82)$ \\
\hline \multicolumn{4}{|l|}{$B R A F, n(\%)$} \\
\hline$B R A F$ V600E/V600 K & $78(65)$ & $65(66)$ & $13(59)$ \\
\hline Wild type & 34 (28) & $29(30)$ & $5(23)$ \\
\hline Other $^{\mathrm{d}}$ & $3(3)$ & $2(2)$ & $1(5)$ \\
\hline Missing & $5(4)$ & $2(2)$ & $3(14)$ \\
\hline
\end{tabular}

${ }^{a}$ Excluding nine patients with unknown primary tumor

${ }^{b}$ Primary melanoma diagnosed as melanocytic tumor of uncertain malignant potential (MELTUMP) in two patients, confirmed by revision

${ }^{\mathrm{c}}$ Including locoregional recurrences

${ }^{\mathrm{d} O n e}$ inactivating mutation, one p.Leu485Trp mutation, one p.Thr599Dup mutation

AJCC American Joint Committee on Cancer, BRAF B-Raf proto-oncogene, serine/threonine kinase

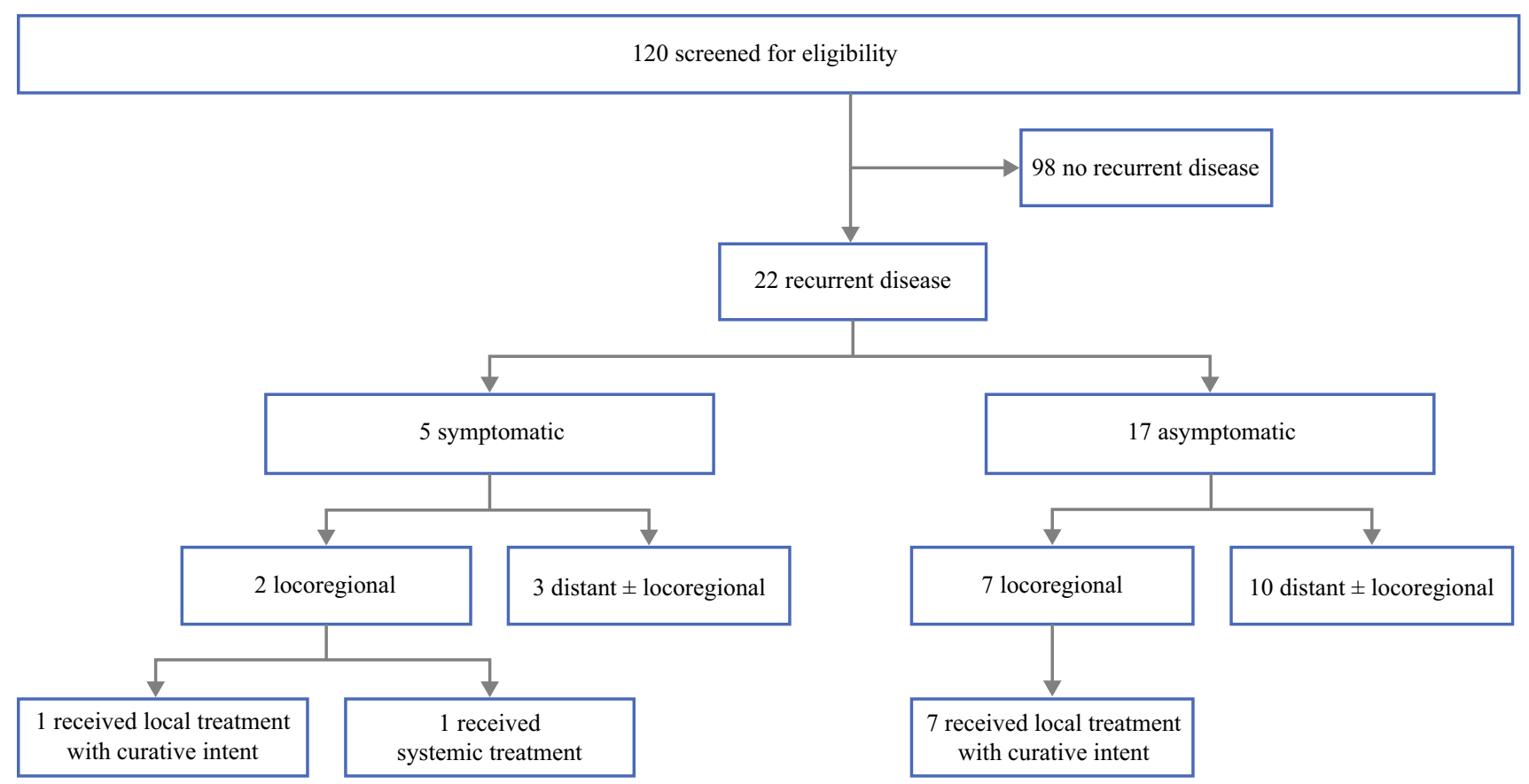

FIG. 1 Detection of recurrent disease during screening for eligibility

patients with and without recurrent disease during screening are presented in Table 1 . No statistically significant differences between groups were present.

\section{Detection of Recurrent Disease}

Melanoma metastasis was detected in $22(18 \%)$ of 120 patients (Fig. 1), corresponding to a number needed to screen of 5.45 to detect one patient with recurrent disease.
Thirteen $(59 \%)$ patients were identified with distant metastasis, while in the remaining nine $(41 \%)$ patients, metastasis was locoregionally located.

Five $(23 \%)$ recurrences were found based on symptoms or physical examination (symptomatic recurrence); in three patients, in-transit metastasis was noticed by the patient $(n=1)$ or physician $(n=2)$, and another patient discovered a local recurrence at the site of the resected primary melanoma. Of these four patients with symptomatic 


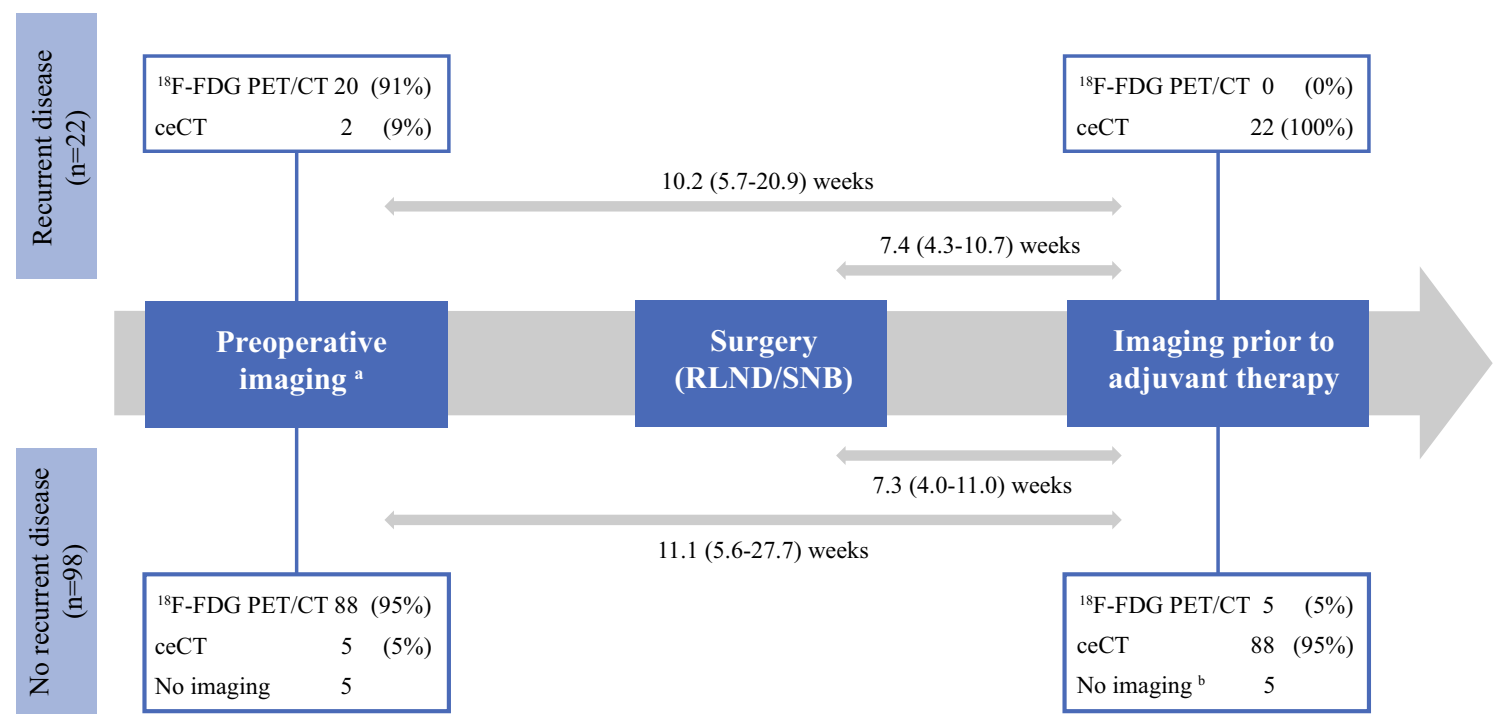

FIG. 2 Time intervals and imaging techniques used prior to intended start of adjuvant therapy. Time intervals presented as median (range). ${ }^{a}$ Imaging prior to referral for trial participation was performed postoperatively after sentinel node biopsy (micrometastatic disease) in nine patients and in three patients with macrometastatic disease.

locoregional relapse, two showed detectable distant metastatic disease on ceCT scan. The fifth patient developed back pain, which was suspicious for bone metastasis and confirmed by ceCT imaging. Seventeen (77\%) relapses were asymptomatic and initially detected by imaging, corresponding to a number of asymptomatic patients needed to screen of 6.76. One of these patients presented with atypical, very small pulmonary nodules before RLND. Another patient showed atypical/nonspecific hypodense liver lesions of maximum $10 \mathrm{~mm}$ on preoperative ceCT scan, and these lesions were identified as liver metastases during screening ceCT after a 12-week interval. Serum LDH level was not a sensitive parameter for recurrent disease, since only $4(18 \%)$ out of 22 relapsed patients had elevated LDH. All four patients had distant metastasis, and two of them were asymptomatic.

\section{Imaging Techniques and Intervals}

Before referral to our trial, metastasis had been excluded with ${ }^{18}$ F-FDG PET/CT $(85 \%)$ or ceCT $(15 \%)$ in 115 patients. Of the five patients in whom metastasis had not been excluded prior to screening, four had resected micrometastasis in the pathology report and one patient had macrometastatic disease. However, in all patients presenting recurrent disease during screening, distant metastasis had been excluded on imaging prior to start of screening for eligibility (Fig. 2). For this group with early relapse, prior imaging was done using ${ }^{18} \mathrm{~F}$-FDG PET/CT in 20 patients $(91 \%)$ and ceCT in the remaining 2 patients.
${ }^{\mathrm{b}}$ Imaging was not repeated during screening for eligibility in five patients, since the inclusion in the adjuvant trial was within 6 weeks after prior imaging. ceCT contrast-enhanced venous phase $\mathrm{CT} ;{ }^{18} \mathrm{~F}$ FDG PET/CT ${ }^{18} \mathrm{~F}$-fluorodeoxyglucose PET scan combined with CT, $R L N D$ radical lymph node dissection, $S N B$ sentinel node biopsy

To screen for eligibility, 110 (96\%) patients had standard ceCT. In the remaining five (4\%) patients, imaging was performed by ${ }^{18} \mathrm{~F}-\mathrm{FDG}$ PET/CT. Relapse during screening was detected by ceCT in all cases. In five patients, imaging was not repeated during screening, since the start of experimental adjuvant therapy was within 6 weeks after prior imaging excluding distant metastasis.

The median interval between imaging during screening and previous imaging was 10.2 (range 5.7-20.9) weeks in recurrent patients. The median interval between complete resection and detection of recurrent disease was 7.4 (range 4.3-10.7) weeks. In patients without recurrent disease, these intervals were not significantly different, with a median interval between scans of 11.1 (range 5.6-27.7) weeks and an interval between resection and imaging of 7.3 (range 4.0-11.0) weeks. Figure 3 shows examples of patients with asymptomatic recurrent disease.

\section{Treatment of Relapsed Patients}

Nine patients showed locoregional metastasis, of whom eight were referred for surgical resection with curative intent. One patient had no evidence of disease after adjuvant radiotherapy, therefore planned surgery was cancelled. This patient was disease free during 13 months of follow-up, then relapsed. Of the seven reoperated patients, six developed recurrent disease. In two of them, distant metastasis occurred within 1 month after resection of the recurrent local disease. In four patients, the interval from resection to recurrent disease was $6,6,8$, and 


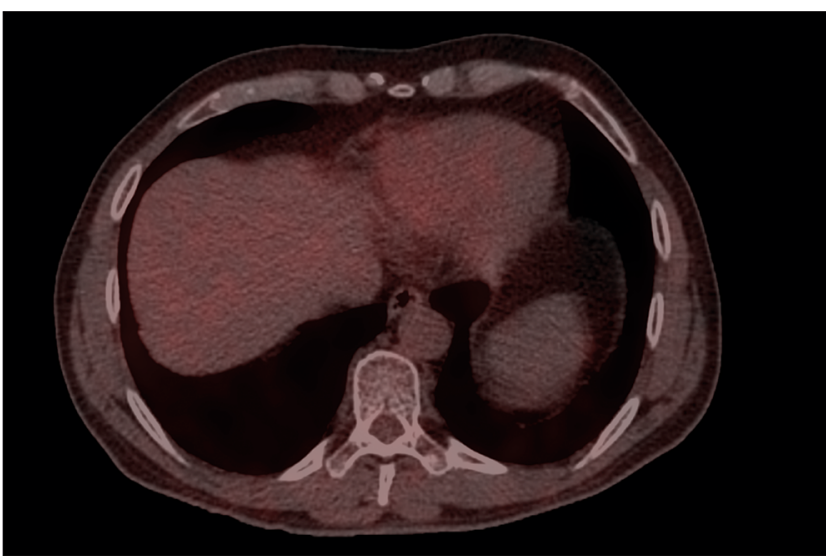

A

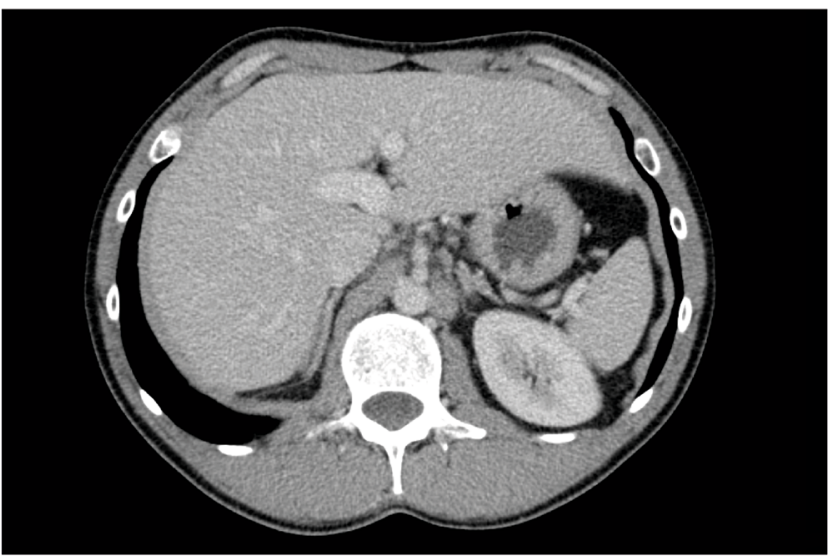

C

FIG. 3 Asymptomatic recurrent melanoma during screening: A patient with pT4aN1b/stage IIIB melanoma (AJCC 7th edition) ${ }^{2}$ showed no metastatic disease on ${ }^{18} \mathrm{~F}$-FDG PET/CT prior to radical lymph node dissection (RLND) (a), but venous-phase contrastenhanced CT (ceCT) 10 weeks after RLND and 12 weeks after prior

9 months. The last reoperated patient is still recurrence free after 10 months of follow-up. In the remaining patient, locoregional recurrence consisted of irresectable in-transit metastasis, for which treatment with anti-PD-1 antibodies was initiated.

Of the 13 patients with distant metastasis, first-line treatment consisted of anti-PD-1 antibodies in three patients, three patients started with combined immune checkpoint inhibition, and in six patients treatment with targeted therapy was initiated. One patient underwent metastasectomy of a solitary liver metastasis.

\section{DISCUSSION}

In 120 patients screened for an adjuvant trial, almost one out of five patients with completely resected stage IIIB or IIIC melanoma showed evidence of recurrent disease prior

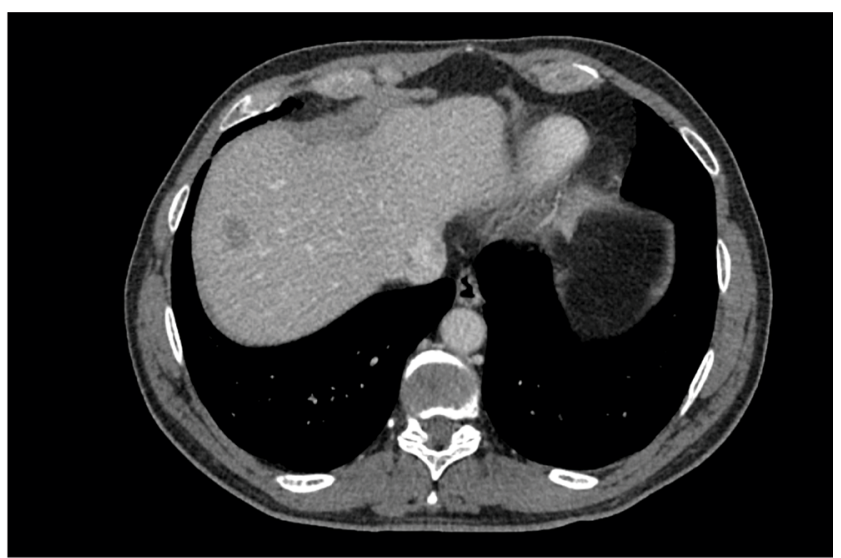

B after 10 weeks

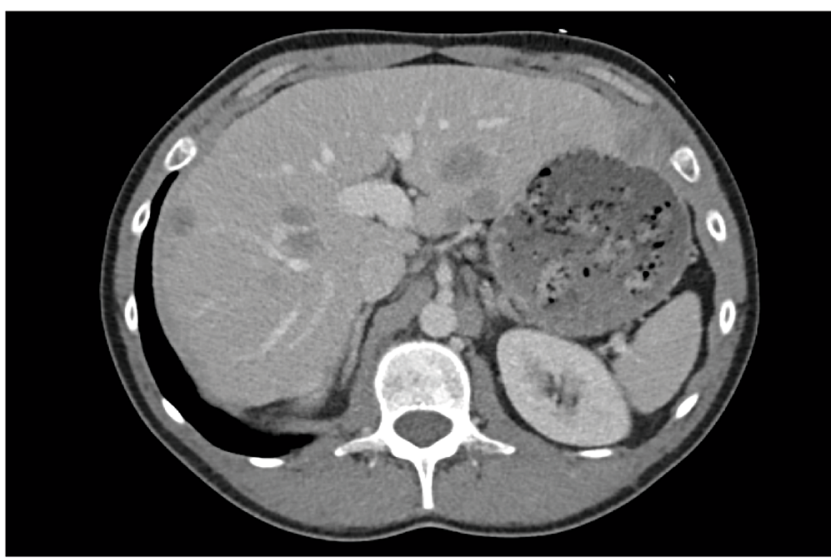

D after 10 weeks

${ }^{18}$ F-FDG PET/CT showed liver metastasis (b); A patient with pT2aN2b IIIB melanoma (AJCC 7th edition) showed no metastatic disease on ceCT (shown) and ${ }^{18}$ F-FDG PET/CT (not shown) prior to RLND (c), but ceCT 5 weeks after RLND and 10 weeks after prior ceCT revealed multiple liver metastases (d)

to start of adjuvant therapy, despite adequate prior imaging. These relapses were present within 2 months after surgery and within 3 months after previous staging. The majority of patients with recurrent disease were asymptomatic, and all were identified by ceCT scan.

Discovery of recurrent disease before start of adjuvant therapy improves information about prognosis. A proper baseline scan prevents incorrectly discarding therapy if metastasis is visualized at the first follow-up scan but was already present and detectable before start of therapy. In addition, evidence of relapse can change therapeutic management. About one-third of patients with recurrent disease were referred for additional resection with curative intent due to locoregional relapse. Furthermore, patients with rapid relapse with relatively high metastatic load started treatment with BRAF/MEK inhibitors or combined anti-CTLA-4/anti-PD-1 antibodies. Therefore, reimaging 
before start of adjuvant therapy leads to a change in therapeutic management in a substantial group of patients and should be considered in all patients despite prior imaging.

A limitation of this study is that we only evaluated patients screened for eligibility in our clinical trial, hence a selection bias might have occurred. Patients with more unfavorable prognosis and higher risk of recurrence are more likely to be referred for trial participation than patients who would be referred for approved adjuvant treatment. On the other hand, some rapid relapses are missed in our report due to development of symptomatic metastasis or due to recurrent disease diagnosed at radiotherapy planning CT scans before screening for eligibility. The interval between scans was similar between the groups with and without relapse, therefore a lead-time bias does not seem to play a role.

To the best of the authors' knowledge, this is the first report about detection of early recurrent disease in resected stage III melanoma before start of adjuvant therapy. Studies have been conducted to analyze the discovery of metastasis by imaging in stage III melanoma patients during follow-up after resection. ${ }^{27-34}$ However, these studies performed imaging during follow-up with a longer interval after surgery and did not report recurrences in relation to start of adjuvant therapy. Mostly, the first scan was conducted 6-12 months after surgery, thus information about rapid asymptomatic relapses within 12 weeks is lacking. In line with our protocol, phase III trials investigating adjuvant treatment with anti-CTLA-4 or anti-PD-1 antibodies or BRAF/MEK inhibitors excluded metastasis with CT postoperatively and within 4-6 weeks prior to randomization. ${ }^{11-14}$ The trial investigating adjuvant ipilimumab versus nivolumab reported that $24 \%$ of screened resected stage IIIB/C/IV patients no longer met criteria and were not randomized. ${ }^{13}$ Exact numbers of screening failures due to recurrent disease were not mentioned but probably represent an important portion thereof. In addition, the contribution of relapse in stage IV melanoma patients, at higher risk for relapse than stage IIIB/C patients, is not reported. It would be interesting to analyze the numbers of recurrent disease during screening in the larger study cohorts of adjuvant phase III trials.

Taken together, about one-fifth of completely resected stage IIIB/C melanoma patients had recurrent disease before start of adjuvant treatment. Because of the impact on prognosis and therapeutic consequences, restaging all high-risk patients before start of adjuvant therapy seems appropriate.

ACKNOWLEDGMENT The authors thank all patients for their interest and willingness to participate in the clinical trial. The authors thank E.F. Smits for data management, S.M. Hins-de Bree for clinical support, and the entire clinical and laboratory team involved in the performance of the trial.
FUNDING This work was supported by NWO Grant 837004014 . I.J.M. de V. received NWO Vici Grant 91814655

\section{COMPLIANCE WITH ETHICAL STANDARDS}

CONFLICT OF INTEREST WR.G. received speaker fees from Astellas, Bayer, Merck Sharp \& Dohme (MSD), and the European Society for Medical Oncology, participated in advisory boards of Amgen, Bayer, Bristol-Myers Squibb, Curevac, Dendreon, IQVIA, Janssen-Cilag, Merck (MSD), Morphosys, and Sanofi, and received research grants from Astellas, Amgen, Bayer, Janssen-Cilaq, and Sanofi. R. H. T. K. has received speaker fees from BMS, MSD, and Roche. He has advisory relationships with BMS, MSD, Novartis, and Roche. He received research grants from BMS and Roche. For the remaining authors, no conflicts of interest are declared.

OPEN ACCESS This article is distributed under the terms of the Creative Commons Attribution 4.0 International License (http://crea tivecommons.org/licenses/by/4.0/), which permits unrestricted use, distribution, and reproduction in any medium, provided you give appropriate credit to the original author(s) and the source, provide a link to the Creative Commons license, and indicate if changes were made.

\section{REFERENCES}

1. Romano E, Scordo M, Dusza SW, Coit DG, Chapman PB. Site and timing of first relapse in stage III melanoma patients: implications for follow-up guidelines. $J$ Clin Oncol. 2010;28(18):3042-47.

2. Balch CM, Gershenwald JE, Soong S-J, et al. Final version of 2009 AJCC melanoma staging and classification. J Clin Oncol. 2009;27(36):6199-06.

3. van Akkooi AC, Bouwhuis MG, van Geel AN, et al. Morbidity and prognosis after therapeutic lymph node dissections for malignant melanoma. Eur J Surg Oncol. 2007;33(1):102-08.

4. Robert C, Thomas L, Bondarenko I, et al. Ipilimumab plus dacarbazine for previously untreated metastatic melanoma. $N$ Engl J Med. 2011;364(26):2517-26.

5. Chapman PB, Hauschild A, Robert $\mathrm{C}$, et al. Improved survival with vemurafenib in melanoma with BRAF V600E mutation. $N$ Engl J Med. 2011;364(26):2507-16.

6. Larkin J, Ascierto PA, Dreno B, et al. Combined vemurafenib and cobimetinib in BRAF-mutated melanoma. $N$ Engl $\mathrm{J}$ Med. 2014;371(20): 1867-76.

7. Long GV, Stroyakovskiy D, Gogas H, et al. Dabrafenib and trametinib versus dabrafenib and placebo for Val600 BRAFmutant melanoma: a multicentre, double-blind, phase 3 randomised controlled trial. Lancet. 2015;386(9992):444-51.

8. Robert C, Schachter J, Long GV, et al. Pembrolizumab versus ipilimumab in advanced melanoma. $N$ Engl $J$ Med. 2015;372(26):2521-32.

9. Robert C, Long GV, Brady B, et al. Nivolumab in previously untreated melanoma without BRAF mutation. $N$ Engl $J$ Med. 2015;372(4):320-30.

10. Larkin J, Chiarion-Sileni V, Gonzalez R, et al. Combined nivolumab and ipilimumab or monotherapy in untreated melanoma. $N$ Engl J Med. 2015;373(1):23-34.

11. Eggermont AM, Chiarion-Sileni V, Grob JJ, et al. Prolonged survival in stage III melanoma with ipilimumab adjuvant therapy. N Engl J Med. 2016;375(19):1845-55.

12. Long GV, Hauschild A, Santinami M, et al. Adjuvant dabrafenib plus trametinib in stage III BRAF-mutated melanoma. $N$ Engl $J$ Med. 2017;377(19):1813-23. 
13. Weber J, Mandala M, Del Vecchio M, et al. Adjuvant nivolumab versus ipilimumab in resected stage III or IV melanoma. $N$ Engl $J$ Med. 2017;377(19):1824-35.

14. Eggermont AMM, Blank CU, Mandala M, et al. Adjuvant pembrolizumab versus placebo in resected stage III melanoma. $N$ Engl J Med. 2018;378(19):1789-801.

15. EMEA/H/C/003985 - PSUSA/00010379/201801 - Product information Opdivo. European Medicines Agency. Accessed November 2018.

16. EMA/H/C/002604 - WS/1274 - Product information Tafinlar. European Medicines Agency. Accessed November 2018.

17. EMA/H/C/002643 - WS/1274 - Product information Mekinist. European Medicines Agency. Accessed November 2018.

18. EMA/CHMP/706955/2018 - CHMP post-authorisation summary of positive opinion for Keytrudy (II-47). European Medicines Agency. Accessed November 2018.

19. Full prescribing information Yervoy. Food and Drug Administration. Accessed November 2018.

20. Full prescribing information Opdivo. Food and Drug Administration. Accessed November 2018.

21. Full prescribing information Mekinist. European Medicines Agency. Accessed November 2018.

22. Full prescribing information Tafinlar. Food and Drug Administration. Accessed November 2018.

23. Full prescribing information Keytruda. Food and Drug Administration. Accessed November 2018.

24. Rueth NM, Xing Y, Chiang YJ, et al. Is surveillance imaging effective for detecting surgically treatable recurrences in patients with melanoma? A comparative analysis of stage-specific surveillance strategies. Ann Surg. 2014;259(6):1215-22.

25. Xing Y, Bronstein Y, Ross MI, et al. Contemporary diagnostic imaging modalities for the staging and surveillance of melanoma patients: a meta-analysis. $J$ Natl Cancer Inst. 2011;103(2):129-42.

26. Faries MB, Thompson JF, Cochran AJ, et al. Completion dissection or observation for sentinel-node metastasis in melanoma. N Engl J Med. 2017;376(23):2211-22.
27. Baker JJ, Meyers MO, Frank J, Amos KD, Stitzenberg KB, Ollila DW. Routine restaging PET/CT and detection of initial recurrence in sentinel lymph node positive stage III melanoma. Am J Surg. 2014;207(4):549-54.

28. Podlipnik S, Carrera C, Sanchez M, et al. Performance of diagnostic tests in an intensive follow-up protocol for patients with American Joint Committee on Cancer (AJCC) stage IIB, IIC, and III localized primary melanoma: A prospective cohort study. $J$ Am Acad Dermatol. 2016;75(3):516-24.

29. Madu MF, Timmerman P, Wouters M, van der Hiel B, van der Hage JA, van Akkooi ACJ. PET/CT surveillance detects asymptomatic recurrences in stage IIIB and IIIC melanoma patients: a prospective cohort study. Melanoma Res. 2017;27(3):251-57.

30. Park TS, Phan GQ, Yang JC, et al. Routine computer tomography imaging for the detection of recurrences in high-risk melanoma patients. Ann Surg Oncol. 2017;24(4):947-51.

31. Lewin J, Sayers L, Kee D, et al. Surveillance imaging with FDG$\mathrm{PET} / \mathrm{CT}$ in the post-operative follow-up of stage 3 melanoma. Ann Oncol. 2018;29(7):1569-74.

32. Lim KHJ, Spain L, Barker C, et al. Contemporary outcomes from the use of regular imaging to detect relapse in high-risk cutaneous melanoma. ESMO Open. 2018;3(2):e000317.

33. Podlipnik S, Moreno-Ramirez D, Carrera C, et al. Cost-effectiveness analysis of imaging strategy for an intensive follow-up of patients with American Joint Committee on Cancer stage IIB, IIC and III malignant melanoma. Br J Dermatol. 2018. https://doi. org/10.1111/bjd.16833.

34. Abbott RA, Acland KM, Harries M, O'Doherty M. The role of positron emission tomography with computed tomography in the follow-up of asymptomatic cutaneous malignant melanoma patients with a high risk of disease recurrence. Melanoma Res. 2011;21(5):446-49.

Publisher's Note Springer Nature remains neutral with regard to jurisdictional claims in published maps and institutional affiliations. 